\title{
Upscaling laser-induced periodic surface structures (LIPSS) manufacturing by defocused laser processing
}

\section{Mezera, G.R.B.E. Römer}

M. Mezera, G.R.B.E. Römer, "Upscaling laser-induced periodic surface structures (LIPSS) manufacturing by defocused laser processing," Proc. SPIE 10906, Laser-based Micro- and Nanoprocessing XIII, 109060 U (4 March 2019); doi: 10.1117/12.2510004

SPIE. Event: SPIE LASE, 2019, San Francisco, California, United States 


\title{
Laser-induced Periodic Surface Structures (LIPSS) created by defocused laser processing
}

\author{
M. Mezera ${ }^{\mathrm{a}}$ and G.R.B.E. Römer ${ }^{\mathrm{a}}$ \\ ${ }^{a}$ University of Twente, Faculty of Engineering Technology, Surfaces and Systems (MS3), Chair \\ of Laser Processing, Drienerlolaan 5, 7522 NB Enschede, The Netherlands
}

\begin{abstract}
Low spatial frequency Laser-induced Periodic Surface Structures (LSFL) have been created on single crystal silicon with picosecond laser pulses with a wavelength of $\lambda=1030 \mathrm{~nm}$ with varying laser spot diameters obtained by a defocused laser beam. The laser processing parameters have been adjusted theoretically and experimentally to obtain similar LSFL for all studied laser spot diameters. The periodicity and amplitude of the LSFL were measured by SEM and AFM analysis. It has been found that the periodicities of the LSFL do not change when LSFL were created with larger laser spot diameters. The amplitudes of the LSFL decrease with increasing laser spot diameters, although this correlation is not strong.
\end{abstract}

Keywords: Laser-induced Periodic Surface Structures (LIPSS); Low spatial frequency Laser-induced Periodic Surface Structures (LSFL); Upscaling; Defocused Processing; LIPSS Periodicity; LIPSS Amplitude

\section{INTRODUCTION}

Processing of solids near their ablation threshold by high intensity polarized laser irradiation may lead to the development of regular nanoscale structures known as Laser-induced Periodic Surface Structures (LIPSS). ${ }^{1}$ Applying LIPSS to a surface can lead to numerous surface functionalisations such as improved wetting, ${ }^{2-5}$ improved tribological properties, ${ }^{4,6}$ optical applications, ${ }^{4,5,7,8}$ anti-bacterial surfaces,${ }^{9,10}$ tissue-engineering, ${ }^{11}$ microfluidics $^{12}$ and optolectronics. ${ }^{13}$ The morphology and dimensions (spatial periodicity and amplitude) of LIPSS are controlled by several laser parameters, including the wavelength $\lambda$, the polarization of the laser light, the angle of incidence of the laser beam relative to the surface of the substrate, the laser peak fluence $F_{0}$ and the fluence distribution profile, the number of laser pulses $N$ and the spatial pulse-to-pulse overlap. ${ }^{14,15}$ LIPSS are typically produced by scanning the surface of the substrate by a focused laser beam. ${ }^{5,7,8,16-20}$ When the focal diameter is small, the creation of LIPSS is a relatively slow process, which limits the production of LIPSS on large surfaces at industrial repetition rates. Therefore it is a necessity to increase the production speed of LIPSS.

In general, LIPSS are processed in the focus of the laser beam, at which the spot size is the smallest and the fluence is the highest. A method to speed up the production of LIPSS could be to increase the spot size of the laser beam on the surface. Optical elements in the beam path can increase the focal spot size of the focused laser beam, as e.g. decreasing the beam size with a beam reducer before the beam enters the focusing lens system, or increasing the focal length by changing the focusing lens. To increase the speed of production without changing the laser system setup, a method to increase the beam spot size on the surface can be achieved by processing the surface with a defocused laser beam. It was shown, the the radii of different types of LIPSS structures, depending on the locally absorbed fluence of the gaussian beam profile, increase with an increased distance of the sample surface from the focal spot. ${ }^{21}$

In this study, upscaled manufacturing of so-called Low Spatial Frequency LIPSS (LSFL) over areas on silicon wafers with a defocused laser beam is demonstrated and compared to processing using a focused laser beam. For the purpose of this study the diffraction grating effect of LIPSS, ${ }^{22}$ serves as a measure to assess the quality of the LIPSS. A diffraction grating consists of parallel grooves having a constant periodicity. The grating diffracts impinging white light into several light beams of different wavelengths at various angles. ${ }^{23}$ When viewed from

Further author information: (Send correspondence to M. Mezera)

M. Mezera: E-mail: m.mezera@utwente.nl, Telephone: +31 534894341

Laser-based Micro- and Nanoprocessing XIII, edited by Udo Klotzbach, Akira Watanabe,

Rainer Kling, Proc. of SPIE Vol. 10906, 109060U · @ 2019 SPIE

CCC code: $0277-786 X / 19 / \$ 18 \cdot$ doi: $10.1117 / 12.2510004$

Proc. of SPIE Vol. 10906 109060U-1 
a given angle at the sample, typically only one wavelength is visible to the human eye. This colorization is usually referred to as a structural color. LSFL can also act as a diffraction grating, but imperfections (in terms of regularity) of the grating lead to greying out of structural colors. LIPSS usually appear after several pulses at a fluence regime near the ablation threshold of the processed material. ${ }^{14}$

It should be noted that creating uniform areas of LSFL is not required for the grating effect to occur. Uniform areas of LIPSS are defined here as areas larger than the area of one laser spot size on the surface of the sample, which are covered completely with the same type of LIPSS. The aim of this paper is to study the periodicities and amplitudes of LSFL when processed as a function of the diameter of the (de-)focused laser spot. To that end, the periodicities and amplitudes of LSFL processed in focus are compared to LSFL processed when defocusing the laser beam while ensuring similar laser processing parameters (fluence and pulse-to-pulse overlap at constant pulse frequency) in order to achieve comparable LSFL, meaning structural colors are visible when using a white light microscope. In this paper, the characteristics of LSFL were studied, by positioning the sample surface in the focus, as well as much as 4 Rayleigh lengths above and below the focus. Finally when changing the beam diameter, once the laser fluence regime is known for which LSFL and structural colors appear on the sample, the laser power has to be adjusted to reach the corresponding fluence levels to create LIPSS.

\section{THEORY}

To gain similar LSFL when processsing with a defocused laser beam and a focused beam, the processing scanning velocity and laser fluence have to be adjusted according to the changing beam diameter. The pulse frequency is set constant. The spatial pulse-to-pulse overlap is a critical parameter, when aiming at creating homogeneous areas of LIPSS. This overlap $O L$ is defined here as: $O L=1-\frac{v}{d}$, see Figure 1b,c. To ensure a constant overlap $O L$, when changing the beam diameter, the pulse frequency $f_{\mathrm{F}}$ and/or the beam scanning velocity need to be adjusted proportionally. The beam diameter $d[\mathrm{~m}]$ (see Figure 1a) along the optical axis $z$ follows from: ${ }^{24}$

$$
d(z)^{2}=d_{0}^{2}+\theta^{2}\left(z-z_{0}\right)^{2}
$$

where $d_{0}$ is the diameter in focus, $\theta[\mathrm{rad}]$ is the full far-field divergence angle of the focused beam, and $z_{0 f}[\mathrm{~m}]$ the location of the focus relative to the origin of the reference frame. Without loss of generality it can be assumed that the origin of this frame is in the focus - i.e. $z_{0}=0$. For a laser beam with a Gaussian intensity profile, the Rayleigh length $z_{\mathrm{R}}[\mathrm{m}]$ is the distance from the focus along the propagation axis of the laser beam, to the location where the diameter of the beam has increased by a factor of $\sqrt{2}$. This implies that, at this location, the area of the cross section of the beam is doubled. ${ }^{25}$

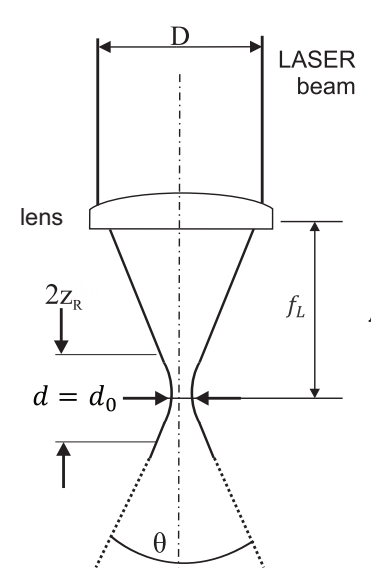

(a) Focusing of Laser beam by a lens

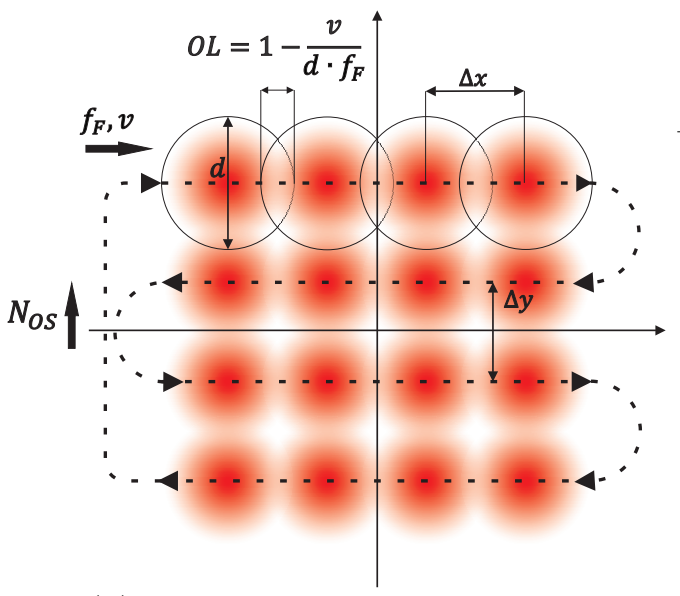

(b) Example of scanning concept

\begin{tabular}{ccc} 
Symbol & Description & Unit \\
\hline$D$ & unfocused beam diameter & {$[\mathrm{m}]$} \\
$z_{\mathrm{R}}$ & Rayleigh Length & {$[\mathrm{m}]$} \\
$d$ & beam diameter along optical axis & {$[\mathrm{m}]$} \\
$d_{0}$ & beam diameter in focus & {$[\mathrm{m}]$} \\
$\theta$ & full far-field divergence angle & {$[\mathrm{rad}]$} \\
$f_{\mathrm{L}}$ & focal length & {$[\mathrm{m}]$} \\
$O L$ & overlap & {$[/]$} \\
$v$ & scanning velocity & {$\left[\frac{\mathrm{m}}{\mathrm{s}}\right]$} \\
$f_{\mathrm{F}}$ & pulse frequency & {$[\mathrm{Hz}]$} \\
$\Delta x$ & horizontal displacement of pulses & {$[\mathrm{m}]$} \\
$\Delta y$ & vertical displacement of pulses & {$[\mathrm{m}]$} \\
$N_{\mathrm{OS}}$ & number of overscans & {$[/]$}
\end{tabular}

(c) Definition of symbols

Figure 1. Schematic representations of the laser beam scanning trajectory and definition of corresponding laser processing parameters 


\section{MATERIALS AND METHODS}

\subsection{Laser Setup and material}

A pulsed Yb:YAG disk laser source (TruMicro 5050 of Trumpf GmbH, Germany) with a wavelength of $1030 \mathrm{~nm}$, maximum pulse frequency of $400 \mathrm{kHz}$, pulse energies up to $125 \mu \mathrm{J}$ and a pulse duration of $6.7 \mathrm{ps}$ was used. The beam was focused with a telecentric F-theta objective (Ronar of Linos GmbH, Germany) having a focal length of $80 \mathrm{~mm}$ on the surface of n-type doped, $\langle 100\rangle$ oriented, single crystal, optical grade silicon wafer samples. The samples were cleaned before the laser processing in an ultrasonic bath for 5 minutes at room temperature with industrial ethanol. The beam was scanned over the sample with a galvoscanner with an accuracy of $2 \mu \mathrm{rad}$ (intelliSCAN14 of ScanLab GmbH, Germany).

\subsection{Analysis tools}

The focal $\frac{1}{e^{2}}$ beam diameter $d_{0}=35.5 \pm 1.61 \mu \mathrm{m}$, Rayleigh length $z_{\mathrm{R}}=857 \pm 39 \mu \mathrm{m}$, full divergence angle $\theta=0.041 \mathrm{rad}$, and the beam quality factor $M^{2}=1.124$ of the focused laser beam were measured using a laser beam characterization device MicroSpotMonitor of Primes GmbH (Germany).

The laser power was measured using a photodiode power sensor at a measurement uncertainty of $\pm 7 \%$ (S132C of ThorLabs, Germany) connected to a readout unit (PM100A of ThorLabs, Germany).

Structural colors were observed using an optical microscope (VHX 5000 of Keyence, Japan) with a magnification of $50 \times$ at an angle of $50^{\circ}$, see Figure 2 .

The periodicities and amplitudes of LSFL were analyzed using a Scanning Electron Microscope (SEM JSM-7200F of JEOL, Japan) and an Atomic Force Microscope (AFM FlexAFM of Nanosurf, Switzerland).

In order to determine the periodicity of LSFL, $120 \times 90 \mu \mathrm{m}^{2}$ SEM micrographs were analyzed with the help of a 2D Fast Fourier Transform (FFT) algorithm including normalization into the laser wavelength using a MATLAB script. ${ }^{26}$ An example is shown in Figure 3. The algorithm converts the spatial image information (of LSFL) into the frequency domain to allow robust determination of the periodicity of LSFL. Additionally, the script allows filtering to reduce noise and increase the accuracy at which the LSFL periodicity is determined. That is, the periodicity and the corresponding standard deviation were obtained from the thousand strongest data points corresponding to LSFL in the frequency plot. Here, a strong frequency is characterized by a large magnitude in the frequency plot.

AFM mircrographs of $50 \times 50 \mu \mathrm{m}^{2}$ were used to determine the amplitude of the LSFL. To this end one hundred profiles perpendicular to the LSFL were obtained automatically from the AFM micrographs and the height differences between each minimum and maximum peak of adjacent ridges of LSFL and the distances between the ridges were recorded. Noise reduction of peak-to-peak distances and amplitudes, which are much smaller or larger than LSFL, was achieved by filtering.

\section{RESULTS AND DISCUSSION}

Initial experiments showed that when processing the sample using the focused laser beam, LSFL form on the silicon sample at an overlap of $O L=0.6$ at a beam spot diameter of $d_{\mathrm{f}}=35.5 \mu \mathrm{m}$, a repetition rate of $f_{\mathrm{F}}=1000 \mathrm{~Hz}$ and at fluence levels ranging from 0.79 to $0.91 \mathrm{~J} / \mathrm{cm}^{2}$. Figure 2 shows the structural colors of LSFL areas obtained at these conditions. For $O L<0.6$ the LSFL are not well defined over the whole area to form diffraction gratings, For OL $>0.6$ imperfections, as for example bubble like features on top of the ridges of LSFL, lead to greying out of the structutural colors on silicon. 


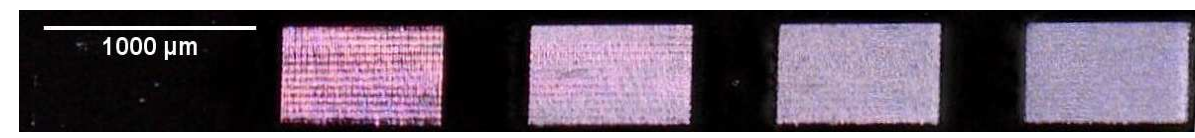

a)

b)

c)

d)

e)

Figure 2. Microscope image of $50^{\circ}$ tilted $750 \times 750 \mu \mathrm{m}^{2}$ areas covered with LSFL obtained by scanning the focused laser beam over the sample at different fluence levels at a constant pulse-to-pulse overlap. From left to right the laser fluence increases in steps of about $0.06 \mathrm{~J} / \mathrm{cm}^{2}$. a) at $0.73 \mathrm{~J} / \mathrm{cm}^{2}$ no structural colors are observed; b) for $0.79 \mathrm{~J} / \mathrm{cm}^{2}$ structural colors are very recognizable; c) at $0.84 \mathrm{~J} / \mathrm{cm}^{2}$ imperfections develop over the LSFL (see also Fig. $5 \mathrm{a}$ and $5 \mathrm{~b}$ ), which leads to greying out of the structural colors; d) at $0.91 \mathrm{~J} / \mathrm{cm}^{2}$ and e) $0.97 \mathrm{~J} / \mathrm{cm}^{2}$ the whole area is covered by imperfections and structural colors are poor.

Next, the beam diameter at up to \pm 4 Rayleigh length distances (RL) from the focus was determined using the beam characterization device. Then, based on these spot diameters and the fluence regimes known to result in LSFL with structural colors (see Figure 2), the scan velocities have been calculated for up to $\pm 4 R L$, see Table 1. Experiments were conducted at a pulse frequency of $1000 \mathrm{~Hz}$ and an overlap of $O L=0.6$ for up to $\pm 4 R L$ in steps of $1 R L$. It was found that the necessary laser fluence needed for achieving LSFL showing structural colors for larger spot diameters is somewhat higher than in focus (see Table 1). This might be explained as follows. When processing an area with ultrashort pulses, every subsequent pulse may lead to accumulation of heat. ${ }^{27}$ The geometrical pulse-to-pulse distance when processed in focus is $14.2 \mu \mathrm{m}$, when processing at $4 \mathrm{RL}$ defocused, the pulse-to-pulse distance equals $58.6 \mu \mathrm{m}$. The thermal diffusion depth ${ }^{28}$ is defined as $d_{\mathrm{t}}=2 \sqrt{\kappa \cdot t_{\mathrm{pp}}}$, where $\kappa=K /\left(\rho \cdot C_{\mathrm{p}}\right)$ is the heat diffusivity, $K$ is the heat conduction coefficient, $\rho$ is the density, $C_{\mathrm{p}}$ is the specific heat of the material and $t_{\mathrm{pp}}=1 / f_{\mathrm{F}}$. When substituting the values for silicon, $K=150 \frac{\mathrm{W}}{\mathrm{mK}},{ }^{29} \rho=2.328 \frac{\mathrm{g}}{\mathrm{cm}^{3}}$, $C_{\mathrm{p}}=0.7 \frac{\mathrm{J}}{\mathrm{gK}} 29$ and pulse frequency of $1000 \mathrm{~Hz}$ applied in the experiments, a thermal diffusion depth of about $600 \mu \mathrm{m}$ is found. Because the geometrical pulse-to-pulse distance in both in-focus and defocused processing is smaller than this thermal diffusion depth, heat accumulation can not be excluded. Since defocused processing has a larger pulse-to-pulse distance than in-focus processing, and less pulses are necessary to structure the $750 \times 750 \mu \mathrm{m}^{2}$ area, less heat accumulates when processing defocused. As the absorptivity of silicon in the infrared rises with increasing temperature, ${ }^{30,31}$ less energy is absorbed when processing defocused when compared to infocus processing. Therefore more energy is needed to achieve structural colors for defocused processing.

Table 1. Laser processing parameters distance to focus position as a function of Rayleigh lengths $R L$, spot diameter $d$, scanning velocity $v$, fluence $F$ and resulting LSFL properties periodicity $\Lambda$ and amplitude $A$. $\Lambda$ and $A$ are taken from the AFM measurements (the indices av and $m$ imply average or median value).

\begin{tabular}{ccccccc}
$R L$ & $d[\mu \mathrm{m}]$ & $v[\mathrm{~mm} / \mathrm{s}]$ & $F\left[\mathrm{~J} / \mathrm{cm}^{2}\right]$ & $\Lambda_{\mathrm{av}}[\mathrm{nm}]$ & $A_{\mathrm{av}}[\mathrm{nm}]$ & $A_{\mathrm{m}}[\mathrm{nm}]$ \\
\hline 0 & 35.5 & 14.2 & 0.79 to 0.91 & $952 \pm 80$ & $206 \pm 88$ & $199 \pm 88$ \\
+1 & 50.3 & 20.1 & 0.78 to 0.83 & $973 \pm 79$ & $173 \pm 89$ & $157 \pm 89$ \\
-1 & 50.3 & 20.1 & 0.89 to 1.03 & $962 \pm 80$ & $204 \pm 85$ & $202 \pm 85$ \\
+2 & 79.5 & 31.8 & 0.84 to 0.88 & $935 \pm 84$ & $115 \pm 69$ & $105 \pm 69$ \\
-2 & 79.5 & 31.8 & 0.88 to 0.93 & $949 \pm 83$ & $127 \pm 66$ & $117 \pm 66$ \\
+3 & 112.4 & 45.0 & 0.91 to 0.98 & $965 \pm 80$ & $122 \pm 59$ & $117 \pm 59$ \\
-3 & 112.4 & 45.0 & 0.98 to 1.02 & $961 \pm 81$ & $138 \pm 76$ & $130 \pm 76$ \\
+4 & 146.6 & 58.6 & 1.13 to 1.14 & $957 \pm 79$ & $86 \pm 66$ & $69 \pm 66$ \\
-4 & 146.6 & 58.6 & 1.13 to 1.14 & $951 \pm 82$ & $106 \pm 53$ & $101 \pm 53$
\end{tabular}




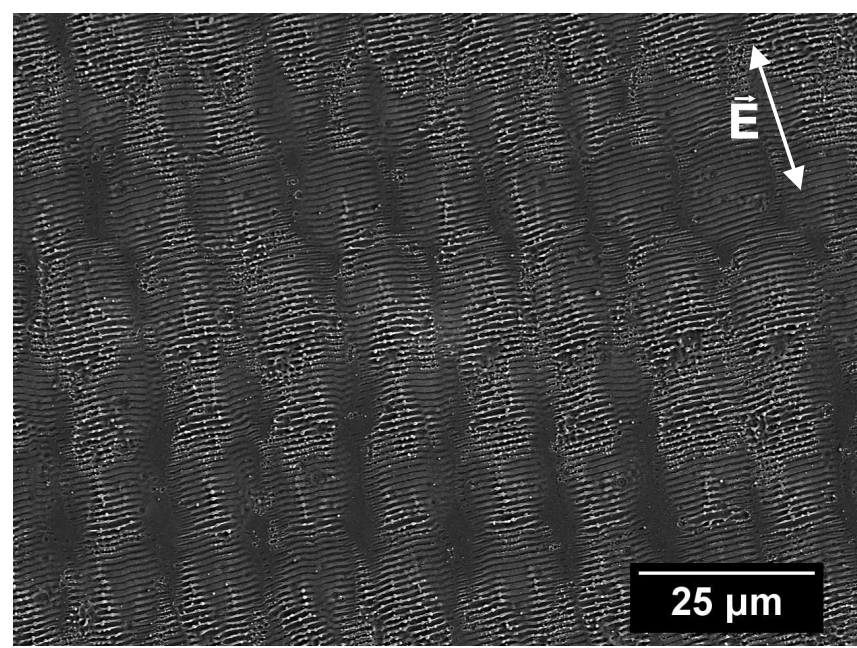

(a) SEM micrograph; the arrow indicates the polarization of the E-field

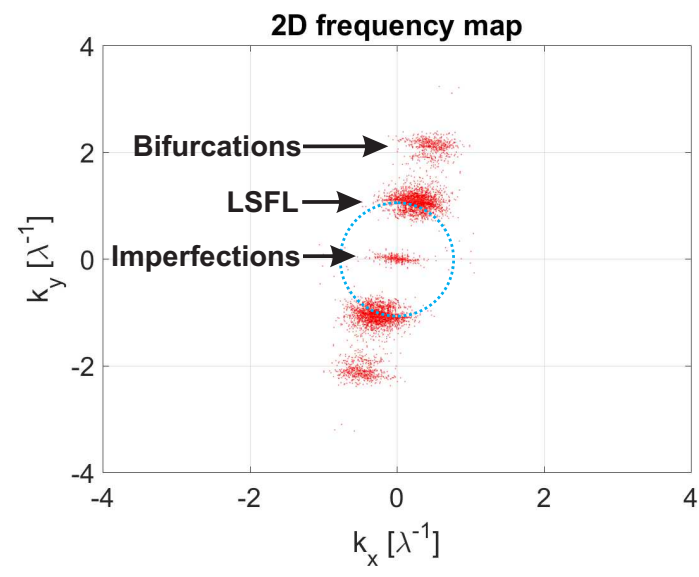

(b) unfiltered 2D frequency map

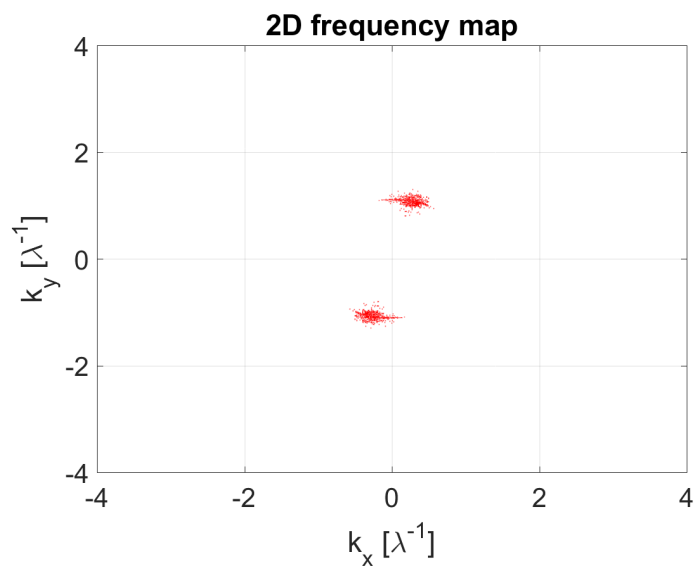

(c) filtered 2D frequency map

Figure 3. a) SEM micrograph; b) and c) frequency analysis of LSFL produced by a focused laser beam $(\mathrm{RL}=0)$ at $0.816 \mathrm{~J} / \mathrm{cm}^{2}, f_{\mathrm{F}}=1000 \mathrm{~Hz}, v=0.014 \mathrm{~m} / \mathrm{s}, O L=0.6$.

Figure 3a shows a SEM micrograph of LSFL obtained while processing the surface of the sample in the focus of the laser beam $(R L=0)$. Figure 3b shows an unfiltered normalized FFT frequency map of the SEM micrograph shown in Figure 3a. The frequency axis were normalized to the laser wavelength $(1030 \mathrm{~nm})$, hence $k_{\mathrm{x}}$ and $k_{\mathrm{y}}$ represent the wavevector in the $x$ - and $y$-direction. The blue dashed circle in Figure $3 \mathrm{~b}$ indicates the wavevector $k=1$. Periodicities in the SEM micrograph are represented by red dots (Figure 3b,3c). Data points outside the circle indicate surface features of a periodicity smaller than the laser wavelength and data points in the circle represent periodicities of features larger than the laser wavelength. In Figure $3 \mathrm{~b}$ three frequencies can be observed. That is, frequencies corresponding to imperfections (bubbles on top of LSFL ridges) found at overlapping areas of LSFL of adjacent pulses; LSFL; and Bifurcations of the LSFL with a periodicity of about half of the LSFL periodicity. After removing imperfections and bifurcations from the frequency map (see Figure $3 c$ ), the periodicity of the LSFL can be easily determined. In that case $930 \pm 40 \mathrm{~nm}$. 


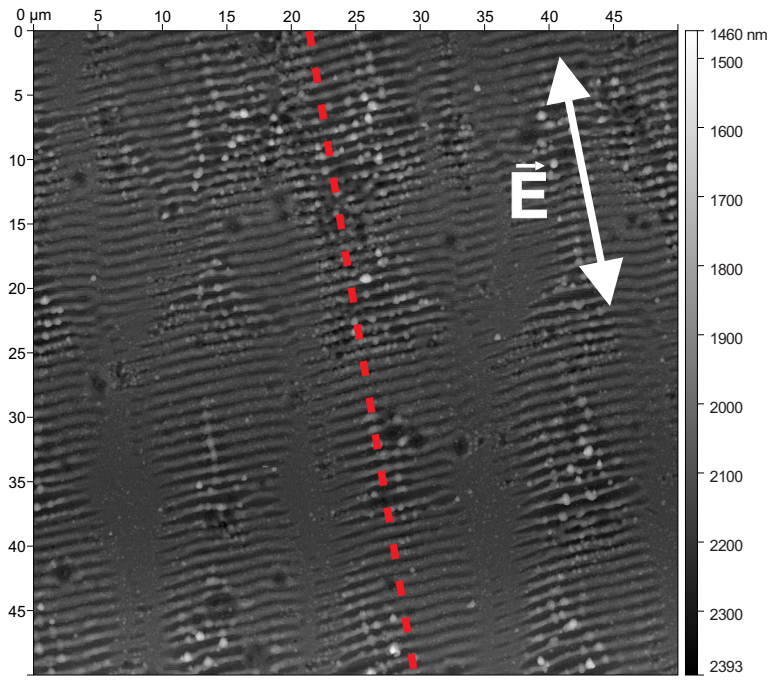

(a) $50 \times 50 \mu \mathrm{m}^{2} \mathrm{AFM}$ micrograph of the surface of the sample processed in focus $(\mathrm{RL}=0)$ at $0.786 \mathrm{~J} / \mathrm{cm}^{2}$, $f=1000 \mathrm{~Hz}, v=0.014 \mathrm{~m} / \mathrm{s}, O L=0.6$; the dashed line indicates the position of one automatically generated profile perpendicular to the LSFL at which the periodicity and amplitude was analyzed; the arrow indicates the polarization direction of the E-field

Figure 4. AFM micrograph and profile

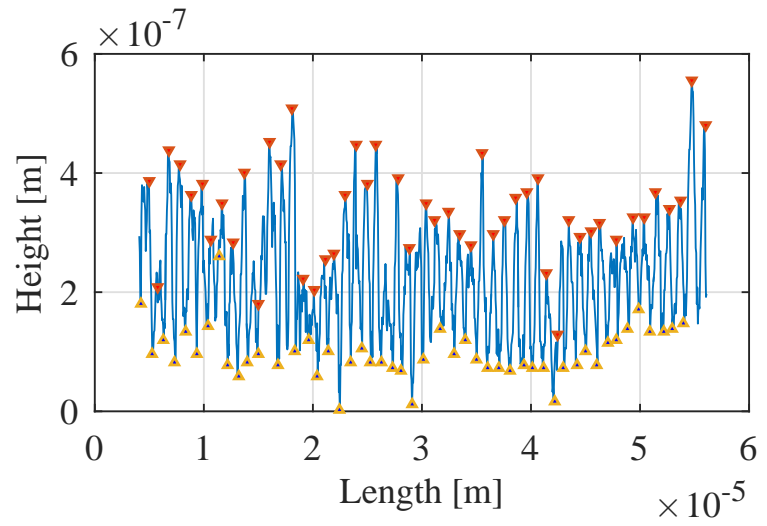

(b) Profile along dashed line in AFM micrograph 4a. The average amplitude was determined by the difference from the maximum peaks (triangles facing downwards) to the adjacent minimum peaks (triangles facing upwards).

Figure 4a shows an example $50 \times 50 \mu m^{2}$ AFM micrograph of the surface of the sample, processed in focus. An automatically generated profile perpendicular to the LSFL along the dashed line in Figure 4a is shown in Figure $4 \mathrm{~b}$. 


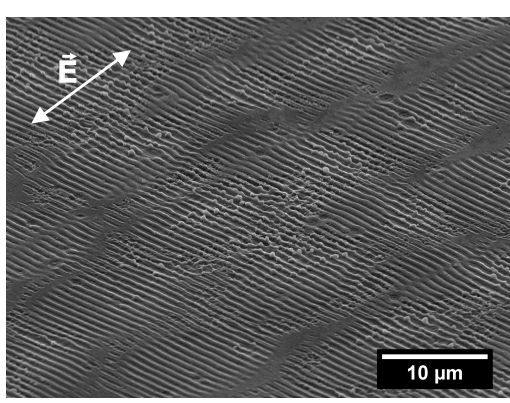

(a) SEM Focus

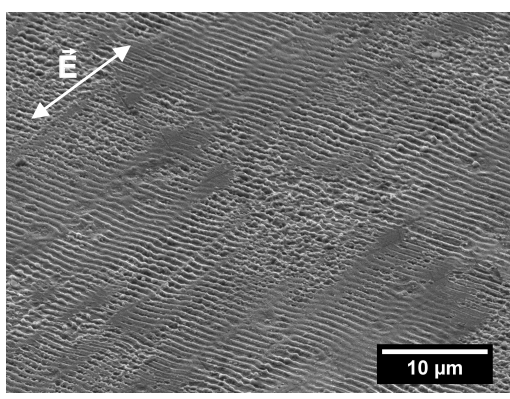

(d) SEM 2RL

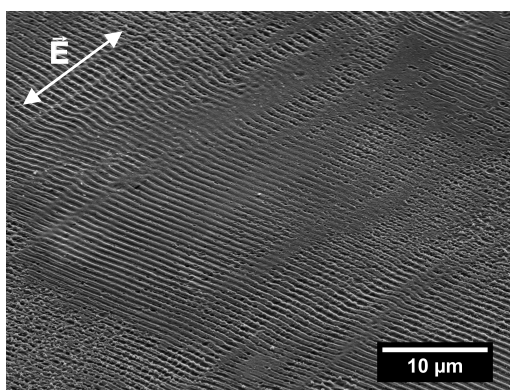

(g) SEM 4RL

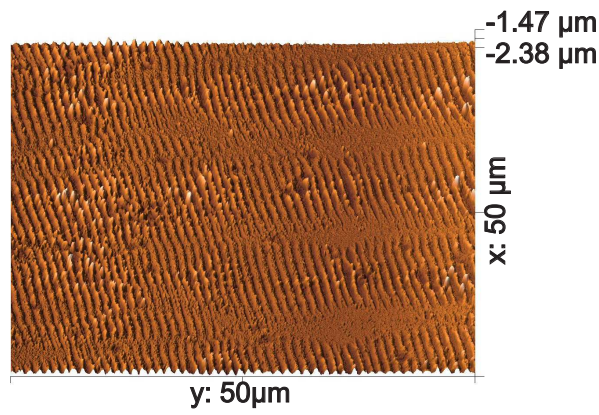

(b) AFM Focus

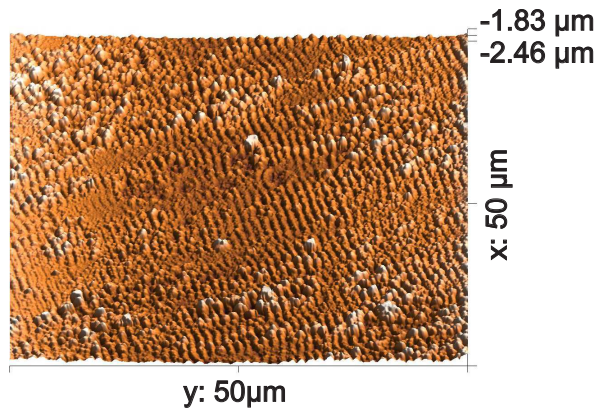

(e) AFM 2RL

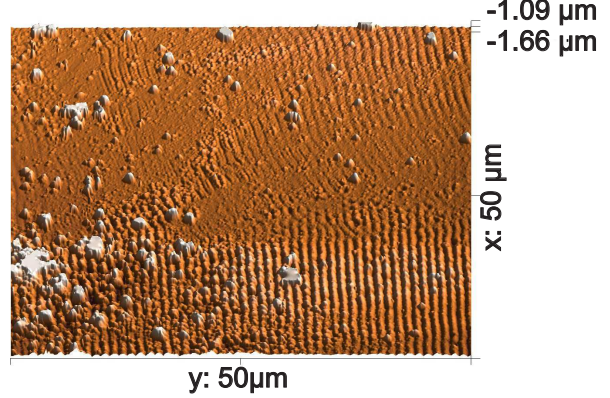

(h) AFM 4RL

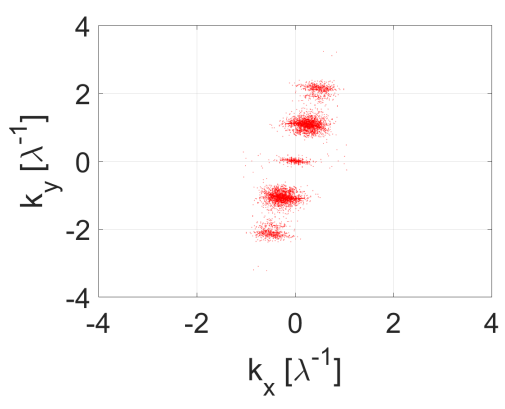

(c) FFT Focus

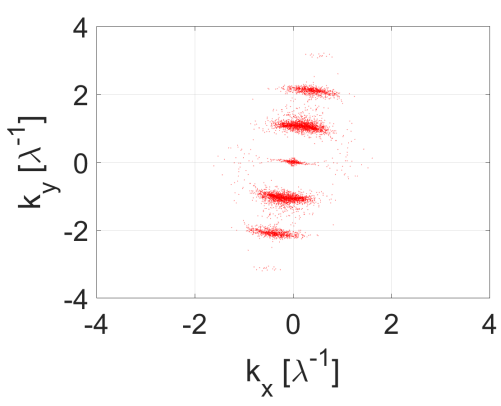

(f) FFT 2RL

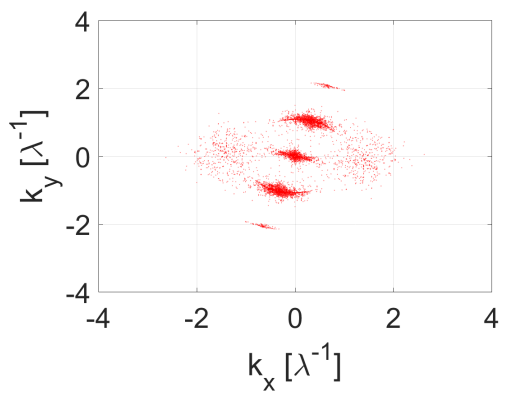

(i) FFT $4 \mathrm{RL}$

Figure 5. Representative micro graphs of LSFL obtained by processing in focus (a,b,c: $\left.F_{0}=0.79 \mathrm{~J} / \mathrm{cm}^{2}\right)$, at 2RL (d,e,f: $\left.F_{0}=0.84 \mathrm{~J} / \mathrm{cm}^{2}\right)$ and at $4 \mathrm{RL}\left(\mathrm{g}, \mathrm{h}, \mathrm{i}: F_{0}=1.13 \mathrm{~J} / \mathrm{cm}^{2}\right)$ above the focus. The graphs (a),(d) and (g) show SEM micrographs taken from tilted samples at an angle of $60^{\circ}$ and a magnification of $2500 \times$; the graphs (b), (e) and (h) show three-dimensional AFM graphs (note that the areas analyzed with the AFM are not the same areas on the sample as the areas analyzed with SEM); the graphs (c), (f) and (i) show the FFT-frequency maps of SEM micrographs. The arrows indicate the polarization direction of the corresponding E-field.

Figure 5 shows representative SEM and AFM micrographs as well as FFT-frequency maps of LSFL obtained by processing in focus, as well as at 2RL and at 4RL above the focus, respectively. As can be observed from Figures $5 \mathrm{a}$ and $5 \mathrm{~b}$, bubble like features develop in areas of LSFL. These features are found at the areas of the intersection of overlapping pulses, as well as at the highest fluence levels of the Gaussian beam profile of the laser spot, see Figure 6. The latter is of course in the direction of the beam path. Huis in 't Veld et al. ${ }^{32}$ also reported bubble formation and claimed that these bubbles develop out of a liquid phase. However, the size of those bubbles $(70-90 \mathrm{~nm})$ are smaller than the size of the bubbles observed in this work (up to 500nm). Therefore, the bubbles in this work might originate from different physical phenomena. The physics of the development of the bubbles falls out of the scope of this paper. When comparing Figure $5 \mathrm{c}$ with Figure 5i, one can observe that the amount of imperfections (bubbles) as well as noise increases. 


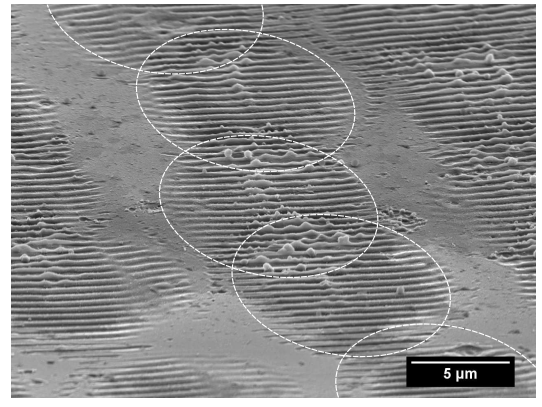

(a) The circles indicate the areas of the laser spot where the laser intensity is above the LSFL threshold. Bubble like features are found at the intersection of overlapping LSFL areas, as well as at the highest fluence levels of the Gaussian beam profile of the laser spot, which are of course in the direction of the beam path (see bubble like features in the center of beampath).

Figure 6. SEM micrographs of the surface processed in focus $\left(\mathrm{RL}=0, F_{0}=0.79 \mathrm{~J} / \mathrm{cm}^{2}\right)$. (a) tilted sample at an angle of $70^{\circ}$; (b) untilted micro graph of same sample.

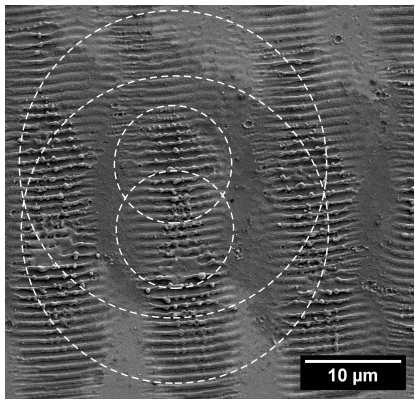

(b) The inner circles indicate the areas of the laser spot where the laser intensity is above the LSFL threshold. The outer circles are indicating the optical beam diameter. One can observe that the bubble like features lie within the optical beam diameter of the adjoining beam path. Interference of the optical energy from the adjoining beam path cannot be excluded.

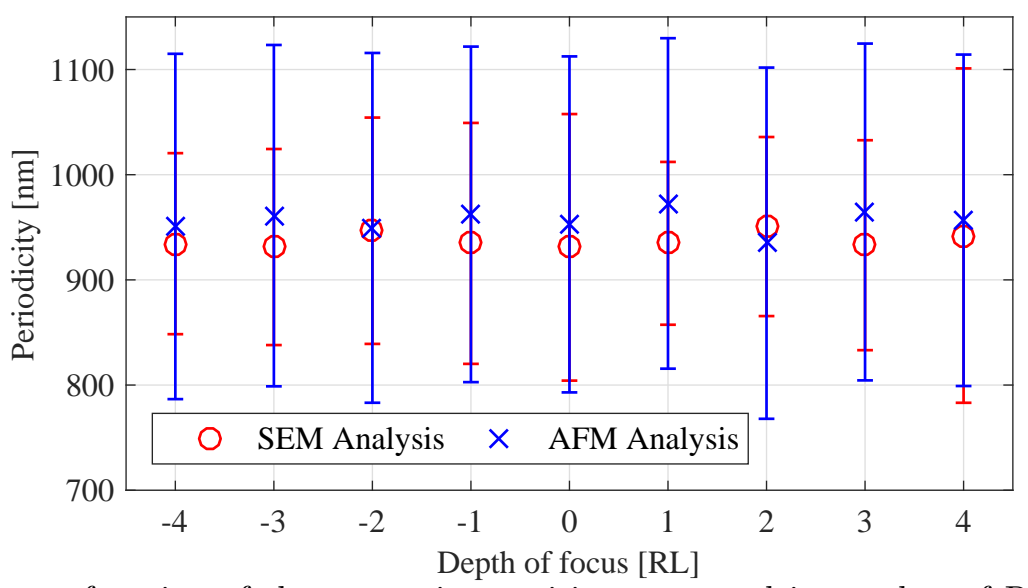

Figure 7. Periodicity as a function of the processing position expressed in number of RL distances above or below the focus obtained from AFM and SEM. The periodicity is constant for all processing positions at about $956 \pm 80 \mathrm{~nm}$. 


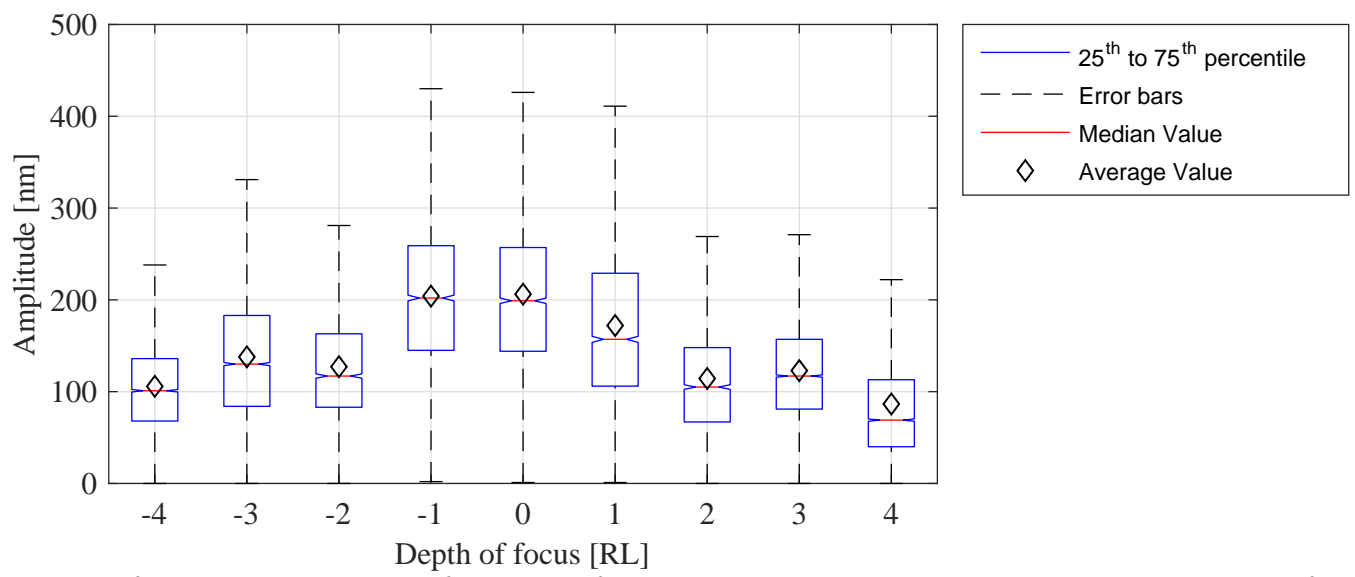

Figure 8. Boxplot of the amplitude as a function of the processing position expressed in number of RL distances above or below the focus obtained from AFM. The notched mark (red) on each box indicates the median value, the diamond indicates the average value and the bottom and top edges of the blue box indicate the 25th and 75th percentiles of the data points, respectively. The dashed verticales indicate the error bars, which are extended up to $1.5 \mathrm{x}$ the interquartile range. ${ }^{33}$

The laser processing parameters and the measured LSFL periodicities and amplitudes for each Rayleigh length distance are listed in Table 1. The periodicity $\Lambda$ and amplitude $A$ of the LSFL are shown graphically in Figures 7 and 8. As can be observed from Figure 7, the periodicities of the LSFL, processed with varying spot diameters, are constant within the measurement accuracy at about $956 \pm 80 \mathrm{~nm}$, which is around the laser wavelength and consistent with literature. ${ }^{14}$ The differences between the SEM and AFM measurements deriving from analyzing disparate areas of the same processed surfaces are negligible and amounts to $\leq 3.3 \%$.

The average values of the amplitudes decrease from around $200 \mathrm{~nm}$, when processing in the focus to about $100 \mathrm{~nm}$ for the largest (around 4 times the focal spot size) used spot diameter. However, when analyzing the average values of the amplitudes in Figure 8, with respect to the statistical errors (error bars), no correlation between the amplitude and the spot diameter can be concluded. On the other hand, when analyzing the boxes of the boxplot (25th and 75th percentiles of the data points), does suggest a correlation between the amplitude and the spot diameter, although this correlation is not strong. This correlation could be explained based on the lower heat accumulation when processing with increasing spot diameter, as was mentioned above. Since there is more heat accumulation when processing in focus, the absorbed energy is higher as when processing defocused. As a result of increased absorbed energy, the LIPSS amplitude increases as was shown in other studies. ${ }^{34-38}$

The geometrical displacements of the individual laser pulses in the $x$ - and $y$ - direction are growing proportionally to the increasing beam diameter. Therefore, the process time increases exponentially with increasing beam diameter. For example processing a $1 \times 1 \mathrm{~cm}^{2}$ area with the laser processing parameters for processing in focus (0RL) will take 7.65 minutes. Four Rayleigh lengths above or below the focus, the processing time will take 29 seconds, dropping to a 16 th of the processing time in focus.

\section{CONCLUSION}

Low spatial frequency LIPSS have been processed on silicon at similar laser processing parameters for varying laser spot diameters by processing the surface of the sample in focus, as well as up to \pm 4 Rayleigh length distances above and below the focus position.

Fixed laser parameters were a pulse duration of $7 \mathrm{ps}$, a laser wavelength of $1030 \mathrm{~nm}$, a pulse frequency of $1000 \mathrm{~Hz}$, a Gaussian beam profile $\left(M^{2}<1.3\right)$. The laser spot diameters ranged from $35 \mu \mathrm{m}$ in focus to $148 \mu \mathrm{m}$ defocused.

The periodicity and amplitude of LSFL were determined from SEM and AFM micrographs. The LSFL showed the same periodicity of $956 \pm 80 \mathrm{~nm}$ for all beam diameters studied. The average amplitude of the LSFL decreases from about $200 \mathrm{~nm}$ to about $100 \mathrm{~nm}$ when increasing the spot diameter from $35.5 \mu \mathrm{m}$ in focus to a diameter of about $146.6 \mu \mathrm{m}$ at $\pm 4 \mathrm{RL}$ distances, although this correlation between amplitude and beam diameter is not strong. 
Defocused laser processing is a simple method for scaling the production rates of LSFL up to industrial rates. Then some basic mathematics show that increasing the spot diameter $d$ while maintaining the geometrical pulseto-pulse overlap $O L$ will decrease the processing time quadratically since both the displacements in the $x$ - and $y$-direction expand proportionally to the increasing spot diameter. For example, using a spot diameter which is 4 times larger than the spot diameter in focus (4RL above the focus), the processing time is only 1/16th (6.25\%) compared to the processing time in focus.

\section{Acknowledgment}

The Laser4Fun project (www.laser4fun.eu) leading to this study has received funding from the European Union's Horizon 2020 research and innovation programme under the Marie Skłodowska-Curie grant agreement No. 675063.

\section{REFERENCES}

[1] Van Driel, H. M., Sipe, J. E., and Young, J. F., "Laser-induced periodic surface structure on solids: a universal phenomenon," Physical Review Letters 49(26), 1955-1958 (1982).

[2] Romano, J.-M., Garcia-giron, A., Penchev, P., and Dimov, S., "Triangular laser-induced submicron textures for functionalising stainless steel surfaces," Applied Surface Science 440, 162-169 (2018).

[3] Kirner, S. V., Hermens, U., Mimidis, A., Skoulas, E., Florian, C., Hischen, F., Plamadeala, C., Baumgartner, W., Winands, K., Mescheder, H., Krüger, J., Solis, J., Siegel, J., Stratakis, E., and Bonse, J., "Mimicking bug-like surface structures and their fluid transport produced by ultrashort laser pulse irradiation of steel," Applied Physics A: Materials Science and Processing 123(12), 1-13 (2017).

[4] Müller, F. A., Kunz, C., and Gräf, S., "Bio-inspired functional surfaces based on laser-induced periodic surface structures," Materials 9(6), 1-29 (2016).

[5] Long, J., Fan, P., Zhong, M., Zhang, H., Xie, Y., and Lin, C., "Superhydrophobic and colorful copper surfaces fabricated by picosecond laser induced periodic nanostructures," Applied Surface Science 311, 461-467 (2014).

[6] Eichstädt, J., Römer, G. R., and Huis in't Veld, A. J., "Towards friction control using laser-induced periodic Surface Structures," Physics Procedia 12(PART 2), 7-15 (2011).

[7] Ionin, A. A., Kudryashov, S. I., Makarov, S. V., Seleznev, L. V., Sinitsyn, D. V., Golosov, E. V., Golosova, O. A., Kolobov, Y. R., and Ligachev, A. E., "Femtosecond laser color marking of metal and semiconductor surfaces," Applied Physics A: Materials Science and Processing 107(2), 301-305 (2012).

[8] Dusser, B., Sagan, Z., Soder, H., Faure, N., Colombier, J., Jourlin, M., and Audouard, E., "Controlled nanostructrures formation by ultra fast laser pulses for color marking," Optics Express 18(3), 2913-2924 (2010).

[9] Lutey, A. H., Gemini, L., Romoli, L., Lazzini, G., Fuso, F., Faucon, M., and Kling, R., "Towards lasertextured antibacterial surfaces," Scientific Reports 8(1), 1-10 (2018).

[10] Kunz, C., Müller, F. A., and Gräf, S., "Multifunctional hierarchical surface structures by femtosecond laser processing," Materials 11(5), 19-26 (2018).

[11] Yiannakou, C., Simitzi, C., Manousaki, A., Fotakis, C., Ranella, A., and Stratakis, E., "Cell patterning via laser micro/nano structured silicon surfaces," Biofabrication 9(2), 025024 (2017).

[12] Paradisanos, I., Fotakis, C., Anastasiadis, S. H., and Stratakis, E., "Gradient induced liquid motion on laser structured black Si surfaces," Applied Physics Letters 107(11), 111603 (2015).

[13] Halbwax, M., Sarnet, T., Delaporte, P., Sentis, M., Etienne, H., Torregrosa, F., Vervisch, V., Perichaud, I., and Martinuzzi, S., "Micro and nano-structuration of silicon by femtosecond laser: application to silicon photovoltaic cells fabrication," Thin Solid Films 516(20), 6791-6795 (2008).

[14] Bonse, J., Höhm, S., Kirner, S., Rosenfeld, A., and Krüger, J., "Laser-induced Periodic Surface Structures (LIPSS) - A Scientific Evergreen," Conference on Lasers and Electro-Optics 23(3), 9000615 (2016).

[15] Buividas, R., Mikutis, M., and Juodkazis, S., "Surface and bulk structuring of materials by ripples with long and short laser pulses: recent advances," Progress in Quantum Electronics 38(3), 119-156 (2014). 
[16] Bizi-Bandoki, P., Benayoun, S., Valette, S., Beaugiraud, B., and Audouard, E., "Modifications of roughness and wettability properties of metals induced by femtosecond laser treatment," Applied Surface Science 257(12), 5213-5218 (2011).

[17] Römer, G. R. B. E., Arnaldo del Cerro, D., Sipkema, R. C. J., Groenendijk, M. N. W., and Huis in't Veld, A. J., "Ultra Short Pulse Laser Generated Surface Textures for Anti-ice Applications in Aviation," in [Proceedings of the 28th International Congress on Applications of Lasers and Electro-Optics, ICALEO], 30-37 (2009).

[18] Ou, Z., Huang, M., and Zhao, F., "Colorizing pure copper surface by ultrafast laser-induced nearsubwavelength ripples," Optics Express 22(14), 17254-17265 (2014).

[19] Vorobyev, A. Y. and Guo, C., "Colorizing metals with femtosecond laser pulses," Applied Physics Letters 92(4), 1-4 (2008).

[20] Eichstädt, J., Römer, G. R. B. E., and Huis in’t Veld, A. J., "Determination of irradiation parameters for laser-induced periodic surface structures," Applied Surface Science 264, 79-87 (2013).

[21] Gregorčič, P., Sedlaček, M., Podgornik, B., and Reif, J., "Formation of laser-induced periodic surface structures (LIPSS) on tool steel by multiple picosecond laser pulses of different polarizations," Applied Surface Science 387, 698-706 (2016).

[22] Emmony, D. C., Howson, R. P., and Willis, L. J., "Laser mirror damage in germanium at $10.6 \mu$ m," Applied Physics Letters 23(11), 598-600 (1973).

[23] Palmer, C., "Diffraction Grating Handbook," Journal of the Optical Society of America 46(1), 20-23 (2005).

[24] Eichler, J., Dünkel, L., and Eppich, B., "Die Strahlqualität von Lasern," Laser Technik Journal 1(2), 63-66 (2004).

[25] Siegman, A. E., [Lasers], University Science Books, Mill Valley, USA (1986).

[26] The MathWorks, I., [MATLABß R2015b], The MathWorks, Inc., Natick, Massachusetts, USA (2015).

[27] Bauer, F., Michalowski, A., Kiedrowski, T., and Nolte, S., "Heat accumulation in ultra-short pulsed scanning laser ablation of metals," Optics Express 23(2), 1035-1043 (2015).

[28] Jee, Y., Becker, M. F., and Walser, R. M., "Laser-induced damage on single-crystal metal surfaces," Journal of the Optical Society of America B 5(3), 648-659 (1988).

[29] Ng, K. K., [Complete Guide to Semiconductor Devices], Wiley-IEEE Press, second edi ed. (2002).

[30] Svantesson, K. G. and Nilsson, N. G., "Determination of the temperature dependence of the free carrier and interband absorption in silicon at 1 e06 pm," Journal of Physics C: Solid State Physics Determination 12, 3837-3842 (1979).

[31] Boyd, I. W., Binnie, T. D., Wilson, J. I. B., and Colles, M. J., "Absorption of infrared radiation in silicon," Journal of Applied Physics 55(8), 3061-3063 (1984).

[32] Huis in 't Veld, B. and van der Veer, H., "Initiation of femtosecond laser machined ripples in steel observed by scanning Helium ion microscopy (SHIM)," Journal of Laser Micro Nanoengineering 5(1), 28-34 (2010).

[33] MathWorks, "Box plot - MATLAB boxplot," (2016).

[34] Ruiz de la Cruz, A., Lahoz, R., Siegel, J., de la Fuente, G. F., and Solis, J., "High speed inscription of uniform, large-area laser-induced periodic surface structures in Cr films using a high repetition rate fs laser," Optics Letters 39(8), 2491-2494 (2014).

[35] Tan, B. and Venkatakrishnan, K., "A femtosecond laser-induced periodical surface structure on crystalline silicon," Journal of Micromechanics and Microengineering 16, 1-6 (2006).

[36] Li, M., Lu, Q. H., Yin, J., Sui, Y., Li, G., Qian, Y., and Wang, Z. G., "Periodic microstructure induced by 532 nm polarized laser illumination on poly(urethane-imide) film: Orientation of the azobenzene chromophore," Applied Surface Science 193, 46-51 (2002).

[37] Rebollar, E., Vázquez De Aldana, J. R., Pérez-Hernández, J. A., Ezquerra, T. A., Moreno, P., and Castillejo, M., "Ultraviolet and infrared femtosecond laser induced periodic surface structures on thin polymer films," Applied Physics Letters 100(4), 041106 (2012).

[38] Rodríguez-Rodríguez, A., Rebollar, E., Soccio, M., Ezquerra, T. A., Rueda, D. R., Garcia-Ramos, J. V., Castillejo, M., and Garcia-Gutierrez, M. C., "Laser-induced periodic surface structures on conjugated polymers: Poly(3-hexylthiophene)," Macromolecules 48(12), 4024-4031 (2015). 